\title{
Sialoadhesin deficiency does not influence the severity of lupus nephritis in New Zealand Black $x$ New Zealand White F1 mice
}

\author{
Dana Kidder ${ }^{1 \dagger}$, Hannah E Richards ${ }^{1 \dagger}$, Paul A Lyons ${ }^{2}$ and Paul R Crocker ${ }^{1 *}$
}

\begin{abstract}
Introduction: Systemic lupus erythematosus (SLE) is a chronic inflammatory condition with multisystem involvement. One of the key features of the disease is the upregulation of type I interferons, resulting in the so-called "interferon signature". Recent flow cytometric and transcriptomic studies identified Sialoadhesin (Sn, CD169) as an important interferon-induced blood monocyte biomarker in diseased patients. To investigate a potential causative role of Sn in SLE, we generated NZBWF1 (New Zealand Black x New Zealand White F1) mice lacking Sn and compared onset and progression of disease with NZBWF1 expressing normal levels of Sn.
\end{abstract}

Methods: Sn expression in renal tissues of pre-diseased and diseased NZBWF1 mice was evaluated by Quantitative real time PCR (QPCR) and immunohistochemistry. $\mathrm{Sn}^{-1-} \mathrm{NZBWF1}$ mice were generated by speed congenics. Disease severity of $\mathrm{Sn}^{+/+}$and $\mathrm{Sn}^{-/-}$NZBWF1 mice was assessed by serum immunoassays, flow cytometry, light microscopy and immunohistochemistry.

Results: Renal tissues from proteinuric NZBWF1 mice exhibited a significant upregulation of Sn mRNA and protein expression following disease onset. Further immunohistochemical analysis showed that $\mathrm{Sn}^{+}$macrophages assumed a distinct periglomerular distribution and, unlike $\mathrm{CD} 68^{+}$macrophages, were not present within the glomeruli. Analysis of disease severity in $\mathrm{Sn}^{-/-}$and $\mathrm{Sn}^{+/+}$NZBWF1 mice revealed no significant differences in the disease progression between the two groups although $\mathrm{Sn}$-deficient mice showed a more rapid onset of proteinuria.

Conclusions: These data confirm a positive correlation of Sn with disease activity. However, Sn deficiency does not have a significant effect on the severity and progression of lupus nephritis in the NZBWF1 mouse model.

\section{Introduction}

Systemic lupus erythematosus (SLE) is an autoimmune chronic inflammatory condition with a multi-system pattern of involvement. SLE is the prototype of systemic autoimmunity of unknown aetiology [1]. The disease is characterized by loss of self-tolerance and accelerated apoptosis with subsequent release of nucleosomal material, which is a major target for immune responses [2]. Failure to clear these apoptotic materials results in expansion of autoreactive lymphocytes, with increased formation of autoantibody [3,4]. Increased levels of antigen-antibody immune-complexes lead to deposition

\footnotetext{
* Correspondence: p.r.crocker@dundee.ac.uk

${ }^{\dagger}$ Equal contributors

${ }^{1}$ Division of Cell Signalling and Immunology, College of Life Sciences, University of Dundee, Dow Street, Dundee DD1 5EH, United Kingdom Full list of author information is available at the end of the article
}

of these in various tissues, causing inflammation and damage by activation of other immune pathways, for example, the complement system. The kidneys are prime sites for immune complex deposition, resulting in glomerulonephritis [5]. Type I IFNs, particularly IFN $\alpha$, were found to be markedly upregulated in patients with active SLE [6]. Interestingly, there was a tendency in the high-IFN group to develop renal, haematological and central nervous system (CNS) disease. These findings confirmed a correlation between this interferon signature and disease severity.

Sialoadhesin, (Sn, CD169, siglec-1) is the prototype of sialic acid-binding immunoglobulin-like lectins (Siglecs) [7]. $\mathrm{Sn}$ is a macrophage-restricted adhesion molecule that is expressed on macrophages of the marginal metalophilic zone and subcapsular sinus in spleen and lymph nodes respectively. $\mathrm{Sn}$ expression can be induced on 
other macrophages under inflammatory conditions, especially under the influence of IFN $\alpha$ [8-11]. Consistent with this observation, Sn was identified as a biomarker for the type I IFN-signature in SLE $[12,13]$. The expression of Sn on blood monocytes was found to correlate with disease severity and other established biomarkers of the disease. However, the role of $\mathrm{Sn}$ in this complex polygenic disease is not clear. The correlation between Sn expression and disease severity suggested a pro-inflammatory role for the molecule. In support of this hypothesis, Sn deficiency was found to ameliorate disease severity in other mouse models of autoimmunity [14-17]. Alternatively, Sn expression might reflect disease activity without a direct role in the disease pathogenesis. The evidence for either argument in SLE is lacking.

These findings prompted us to examine the potential role of $\mathrm{Sn}$ in a well-established murine model of SLE, New Zealand black X New Zealand white F1 (NZBWF1) mice. In this study we examined the expression of $\mathrm{Sn}$ in renal cortical tissues prior to disease onset and in diseased mice. We found that the frequency of $\mathrm{Sn}^{+}$macrophages directly correlated with histological severity. To determine the impact of $\mathrm{Sn}$ in disease pathogenesis, we generated Sn-deficient $\left(\mathrm{Sn}^{--}\right)$NZBWF1 mice using a speed congenics approach. Surprisingly, there was no significant difference in disease severity between wild-type (WT) and Sn-deficient mice. These findings indicate that Sn represents a biomarker for the disease rather than contributing a dominant role in the pathogenesis.

\section{Methods \\ Mice}

New Zealand white (NZW), New Zealand black (NZB) and NZBWF1 mice were purchased from Harlan Laboratories (Oxford, UK) or bred and maintained by Biological Services at the University of Dundee, (Dundee, UK). The generation of Sn-deficient mice was previously reported by our laboratory [18]. The Sn-deficient mice that were initially used to generate $\mathrm{Sn}^{-1-}$ NZBWF1 mice were intercross offspring of heterozygotes backcrossed for more than 20 generations onto a C57BL/6 background. Mice were housed under specific-pathogen-free conditions. The animal protocols used in this study were approved by the Ethical Review Committee of the University of Dundee. All procedures involving living animals were conducted according to the requirement of the United Kingdom Home Office Animals Scientific procedures Act (1986), under PPL60/3856.

\section{Generation of $\mathrm{Sn}^{-/-}$NZBWF1 mice}

The generation of NZBWF1 mice deficient for Sn was done in three phases: (1) generation of parallel NZB and NZW mice that are heterozygous for Sn; (2) crossing Sn heterozygous mice for each strain to generate NZB and
NZW mice wild-type and deficient for Sn; (3) intercrossing WT or Sn-deficient male NZW mice with WT or Sn-deficient NZB mice, to generate $\mathrm{Sn} \mathrm{WT}$ and $\mathrm{Sn}^{-/-}$ NZBWF1 mice.

Speed congenics [19] was employed to transfer a set of predefined NZB and NZW susceptibility loci (Additional file 1) to recipient mice that are heterozygous for Sn. Monitoring the introgression of these loci was examined by genotyping with microsatellite markers (20 for NZB and 25 for NZW).

Ear biopsies from mice in each progeny were genotyped initially for the presence of the Sn mutation. All Sn heterozygous NZB and NZW male mice were then screened for relevant polymorphic markers (Additional file 1). Once all required susceptibility loci were introgressed, further genotyping was done for Sn only. All PCRs were done using the GoTaq ${ }^{\circ}$ Flexi DNA polymerase PCR kit (Promega, Southamptom, UK) using $100 \mathrm{mM}$ 2'-deoxynucleoside 5'-triphosphate (dNTP) purchased from Invitrogen. The primer sets used in genotyping of NZW polymorphic markers were designed using Primer Express software 2.0 (Applied Biosystems, California, USA), also found in Additional file 1.

\section{Quantitative reverse transcription PCR (qRT-PCR)}

RNA extraction was done using RNeasy mini kit (Qiagen, Manchester, UK) according to manufacturer's instructions. In this protocol, renal cortical samples from the left kidney were lysed in the presence of denaturing guanidinethiocyanate-containing buffer to inactivate RNases. Sample homogenization was done with a rotor-stator homogenizer using homogenization tubes containing $1.4-\mathrm{mm}$ ceramic beads (Precelys, Peqlab, Southampton, UK).

The concentration of the extracted RNA was measured based on the absorbance at $260 \mathrm{~nm}$ (A260) in a NanoDrop 1000 spectrophotometer (Thermoscientific, Massachusetts, Boston, USA). The purity of RNA was estimated by measuring the ratio of the readings at $260 \mathrm{~nm}$ and $280 \mathrm{~nm}$ (A260/A280). Sample ratios between 1.9 and 2.1 were regarded as acceptable for CDNA preparation.

cDNA synthesis was performed using a QuantiTect reverse transcription kit (Qiagen). All incubations were done in a thermocycler (Bio-Rad, Hertfordshire, UK) for the specified temperatures and durations. All cDNA samples were stored at $-20^{\circ} \mathrm{C}$ until used in qRT-PCR experiments. cDNA was subsequently quantified with a StepOne Plus detection system (Applied Biosystems, California, USA) using specific primers for each marker and a SYBR Green-based detection system (Applied Biosystems, California, USA). Input RNA was normalised between samples using GAPDH as an endogenous control. Fold upregulation of the target gene was calculated as ratio target gene expression (experimental/ control). Primers used in these experiments were as follows: SnD2 5'-CCCAGCCCCCCCACTAT-3' and 5'-AAGTTCC TCTCCATGCCTTCAC-3'; CD68 5'-CTTCCCACAGGCA 
GCACAG-3' and 5'-AATGATGAGAGGCAGCAAGAG G-3'; IFNaR1 5'-TGGTGGTTCTGTCTCGGTGTT-3' and 5'-GCTACGGCAGGATTAAAAATCG-3'; IFNy 5'-AGCT CTTCCTCATGGCTGTT-3' and 5'-TTTGCCAGTTCCT CCAGATA-3'; IL-1 3 5'-AAGTGATATTCTCCATGAGC TTTG-3' and 5'-TTCTTCTTTGGGTATTGCTTGG-3'; TNF $\alpha$ 5'-CGTCGTAGCAAACCACCAAG-3' and 5'-TTG AAGAGAACCTGGGAGTAGACA-3'; IL-6 5'-TGGGAAA TCGTGGAAATGAG-3' and 5'-CTCTGAAGGACTCTGG CTTTG-3'.

\section{Measurement of proteinuria}

Mice were monitored for disease onset by testing urine with a test strip (Uristix, Bayer, Berlin, Germany) for protein excretion on a weekly basis until proteinuria was detected and then thrice weekly thereafter. Results were graded according to the extent of proteinuria as follows: $0=$ negative or trace, $+=30 \mathrm{mg} / \mathrm{dl},++=100 \mathrm{mg} / \mathrm{dl},+++=300 \mathrm{mg} / \mathrm{dl}$ and $++++=>2,000 \mathrm{mg} / \mathrm{dl}$. Onset of disease was defined as two consecutive readings equal to or more than ++ (100 mg/dl).

\section{Analysis of serum anti-ss/dsDNA}

Sera from BWF1 mice were analysed for IgM and IgG antiss/dsDNA titres using ELISA. Then, 96-well microtitre plates were coated with $20 \mu \mathrm{g} / \mathrm{ml}$ of poly-L lysine (Sigma, Dorset, UK) in deionised water overnight at $4^{\circ} \mathrm{C}$. Plates were washed three times with PBS and $20 \mu \mathrm{g} / \mathrm{ml}$ calf thymus DNA was added for 3 hours at $37^{\circ} \mathrm{C}$. Plates were washed three times with $\mathrm{PBS}+0.05 \%$ Tween (PBST) and nonspecific binding was blocked using PBS $+3 \%$ BSA for 1 hour at $37^{\circ} \mathrm{C}$. Following three washes with PBST, serial dilutions of sera were added in triplicate for 2 hours at $37^{\circ} \mathrm{C}$. Sera dilutions were started at $1 / 100$ to $1 / 6,400$ in PBS $+0.5 \%$ BSA. Plates were washed three times with PBS $+0.5 \%$ BSA. Secondary antibodies diluted in PBS $+0.5 \%$ BSA used for detection of immunoglobulin (Ig)M and IgG anti-ss/dsDNA included alkaline phosphatase-conjugated goat anti-mouse IgM (Sigma, Dorset, UK) or horseradish peroxidase (HRP)conjugated goat anti-mouse IgG (Sigma, Dorset, UK). This was followed by five washes with PBST. Plates were developed either with p-nitrophenyl phosphate substrate (Sigma, Dorset, UK) for alkaline phsophatase-conjugated secondary antibodies or TMB substrate (BD Bioscience, Oxford, UK) for HRP-conjugated secondary antibodies. Plate readings were analysed at $405 \mathrm{~nm}$ for the former and $450 \mathrm{~nm}$ for the latter. Pooled sera from old female NZBWF1 mice were used as a positive control in all experiments.

\section{Flow cytometry}

All staining and washes were carried out in ice-cold fluorescence-activated cell sorting (FACS) buffer (PBS + $0.5 \% \mathrm{BSA}+2 \mathrm{mM}$ ethylenediaminetetraacetic acid (EDTA) $+0.05 \% \mathrm{w} / \mathrm{v}$ sodium azide) and tubes incubated on ice to minimise antibody internalisation. Non-specific binding was limited by incubation with $0.25 \mu \mathrm{g}$ of anti-CD16/CD32 (Fc blocking antibody 2.4G2, eBioscience, San Diego, California, USA) per 1 million cells in $25 \mu$ for 15 minutes. Surface labelling was carried out for 30 minutes with optimal concentrations of the relevant antibodies as decided by prior antibody titrations. The antibodies used in this study include rat anti-mouse; -CD4 PerCp-Cyanine5.5 (clone RM4-5, eBioscience, San Diego, California, USA); -CD8a BD Horizon V500 (clone 53-6.7, BD eBioscience, San Diego, California, USA); -CD19 FITC (clone eBio1D3, eBioscience, San Diego, California, USA); -B220 Alexa Fluor 700 (clone RA3-6B2, eBioscience, San Diego, California, USA), and -CD69 BD Horizon V450 (clone H1.2 F3, BD Biosciences). Cells were washed twice with FACS buffer followed by fixation and permeabilisation using the Foxp3/Transcription Factor Staining Buffer Set (eBioscience, San Diego, California, USA) according to the manufacturer's instructions. Briefly, cells were stained with rat anti-mouse Foxp3 Alexa Fluor 700 (clone FJK-16, eBioscience, San Diego, California, USA) in permeabilisation buffer, washed twice in the same buffer and resuspended in FACS buffer. Samples were then analysed with a BD LSR Fortessa (BDBiosciences, University of Dundee, Central Services) followed by FlowJo version 7.6.4.

\section{Renal histology and assessment of disease severity}

The right kidney was used for assessing lupus nephritis severity by light microscopy. Kidneys were fixed in $10 \%$ neutral-buffered formalin. Processing and staining of kidney sections was conducted by the Veterinary Services, University of Glasgow, UK. Kidney sections $(5 \mu \mathrm{m})$ were stained with H\&E or Masson's trichrome and evaluated by light microscopy. Scoring of nephritis severity included examining glomeruli, tubular and perivascular regions, as described previously [20]. Glomerular pathology was assessed by counting the number of cells per glomerular cross-section (gcs), of 30 glomeruli per kidney. The scoring system was as follows; score 0 , normal cellularity (35 to 40 cells/gcs); 1 , mild hypercellularity (41 to 50 cells/gcs); 2, moderate hypercellularity (51 to 60 cells/gcs) with segmental or diffuse proliferative changes; 3 , severe hypercellularity (>60 cells/gcs) with segmental or global sclerosis, crescent formation and severe exudation. Tubular pathology was assessed based on the percentage of tubules showing dilatation or atrophy among 200 tubules in a randomly chosen field. Perivascular cell accumulation was also evaluated by counting the number of cell layers surrounding randomly chosen inter- and intralobular arteries. Perivascular scoring included; 0 , no perivascular infiltrate; 1 , less than 5 layers surrounding less than half the diameter of the artery; 2, 5 to 10 layers surrounding more than half of the diameter of the vessel; 3, more than 10 layers surrounding more than half the diameter of the artery). Scoring of all kidney sections was done in a blinded fashion. 


\section{Immunohistochemistry}

Kidneys were snap-frozen and stored at $-80^{\circ} \mathrm{C}$ until sectioned: $5-\mu \mathrm{M}$ cryostat sections were prepared, air-dried and fixed in acetone for 10 minutes. Rat anti-mouse monoclonal antibodies against Sn (clone SER4 and 3D6, in-house), CD68 (clone FA-11, eBioscience, San Diego, California, USA), CD4 (clone L3T4, eBioscience, San Diego, California, USA) and Foxp3 (clone FJK-16, eBioscience, San Diego, California, USA) were used to assess the presence of different cell populations. HRPlabelled goat anti-rat Ig was used as secondary antibody (mouse-adsorbed; Vector laboratories, Peterborough, UK). Spleen tissues from Sn wild-type and deficient mice were used as positive and negative controls for $\mathrm{Sn}$, respectively.

\section{Statistical analysis}

Statistical significance was determined by the MannWhitney test. A P-value was considered significant when less than 0.05 .

\section{Results}

Sn expression is upregulated in renal tissue of diseased NZBWF1 mice

Recent analyses of $\mathrm{Sn}^{+}$monocytes in patients with active SLE showed an increase in their frequency that matched disease activity $[12,13]$. However, it is unclear whether $\mathrm{Sn}$ expression is upregulated on macrophages in diseased renal tissues. To this end, we examined the expression of

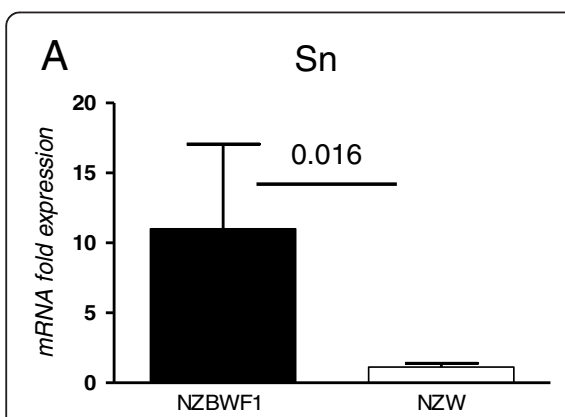

B

Pre-Disease, $\mathrm{Sn}$

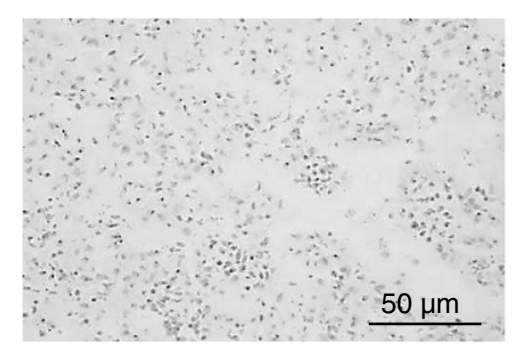

C

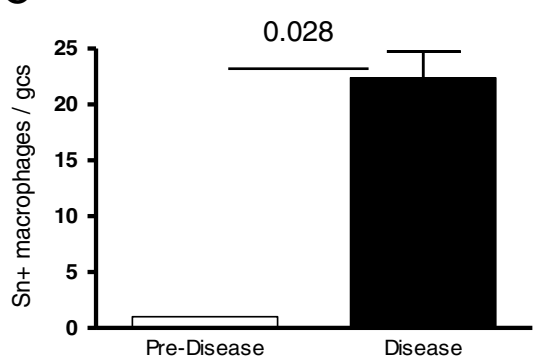

CD68

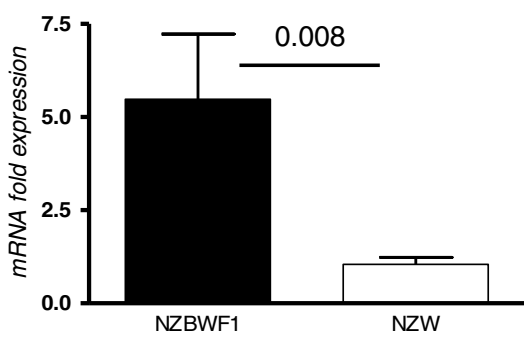

Disease, $\mathrm{Sn}$

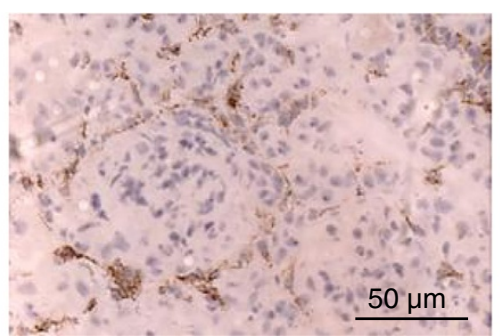

$\mathrm{F} 4 / 80$

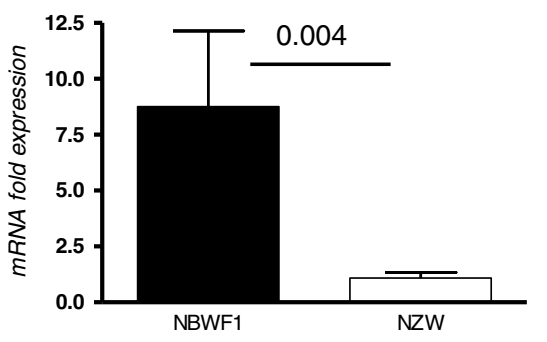

Disease, CD68

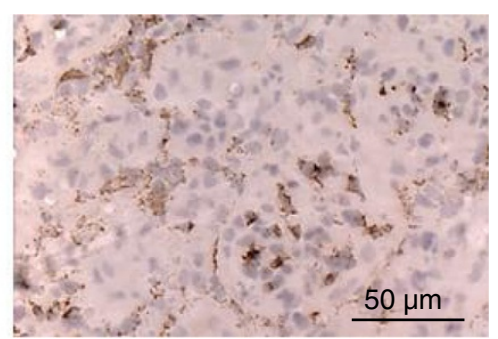

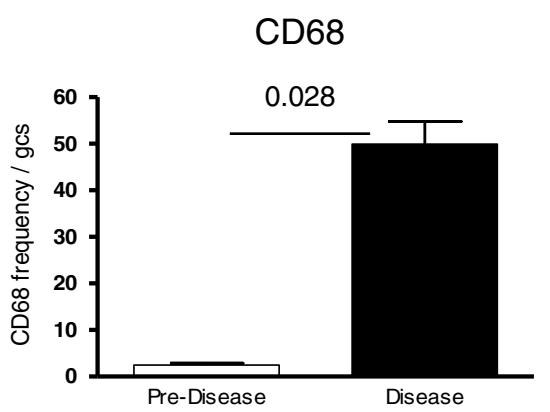

Figure 1 Upregulation of renal sialoadhesin (Sn) expression following disease onset in BWF1 mice. (A) Renal cortical tissues from 28-week proteinuric BWF1 mice and age -atched NZW mice were examined for gene expression of Sn, CD68 and F4/80 ( $n=4$ per group) by qRT-PCR. (B) Immunohistochemistry of frozen kidney sections from pre-disease (10 weeks) and diseased (28 weeks) NZBWF1 mice, indicating the anatomical locations of $\mathrm{CD} 8^{+}$and $\mathrm{Sn}^{+}$macrophages in renal tissues of diseased NZBWF1 mice. (C) Quantification of $\mathrm{Sn}^{+}$macrophages in glomerular crosssections (gcs). Statistical analysis was done using the Mann-Whitney test and $P$-values are indicated. 
Sn and pan-macrophage lineage markers CD68 and F4/80 in renal tissues of 28 week old proteinuric NZBWF1, age matched NZW and pre-disease NZBWF1 mice by qRTPCR. This revealed that dramatic upregulation in the mRNA expression of Sn, CD68 and F4/80 occurs in proteinuric NZBWF1 mice (Figure 1A). There were no significant differences between pre-disease NZBWF1 and control NZW mice (data not shown). In order to confirm the expression of $\mathrm{Sn}$ at a protein level, we used immunohistochemical staining for Sn and CD68 in renal tissues from NZBWF1 mice. Consistent with the qRT-PCR analysis, Sn was absent in pre-diseased kidneys and increased in renal tissues of diseased NZBWF1 mice (Figure 1B and C). Interestingly, $\mathrm{Sn}^{+}$macrophages assumed a distinct periglomerular and tubulointerstitial distribution and, in contrast to $\mathrm{CD} 68^{+}$macrophages, were not found within the glomerular capillaries (Figure 1B).

Concomitant with upregulation of $\mathrm{Sn}$ in diseased renal tissues of NZBWF1 mice, we also observed increased expression of the Ifnarl gene (Figure 2A), similar to what has been reported previously in lupusprone MRL/lpr mice as part of the interferon signature [21]. In addition, genes encoding several proinflammatory cytokines were found to be upregulated in their expression, including IFN $\gamma$, TNF $\alpha$, IL- $1 \beta$ and IL-6 (Figure 2). Taken together, these data show that $\mathrm{Sn}$ is upregulated in kidney tissues from nephritic
NZBWF1 mice and this is associated with enhanced expression of a number of inflammatory cytokines.

\section{Sialoadhesin deficiency does not affect the severity of lupus nephritis in NZBWF1 mice}

To investigate whether the strong upregulation of $\mathrm{Sn}$ is important in the pathogenesis of lupus nephritis, we generated Sn-deficient NZBWF1 mice as described in Methods. Sn deficiency was confirmed by means of PCR (data not shown) and immunohistochemistry of spleen and kidney sections (Figure 3). Age-matched $\mathrm{Sn}^{+/}$, $\mathrm{Sn}^{+/-}$and $\mathrm{Sn}^{-1-}$ mice were monitored for disease onset (proteinuria) and progression leading to death (Figure 4A). No significant differences were observed. However, 3 $\mathrm{Sn}^{-1-}$ mice showed early proteinuria at weeks 16, 17 and 21 , which subsided and then reappeared at weeks 21,25 and 31 respectively, progressing to death. A fourth $\mathrm{Sn}$-deficient mouse developed early proteinuria at week 21 which progressed. The earliest onset of proteinuria in Sn-expressing mice was at week 23 and in all cases, disease was progressive (Figure 4A). This difference in the early onset of proteinuria between Sn-deficient mice and Sn-expressing mice was significant (Fishers exact test, $P=0.04$ ).

The analysis of serial serum samples from $\mathrm{Sn}^{+/+}$ and $\mathrm{Sn}^{-1-}$ NZBF1 mice for IgM and IgG anti-dsDNA showed no significant differences between the two
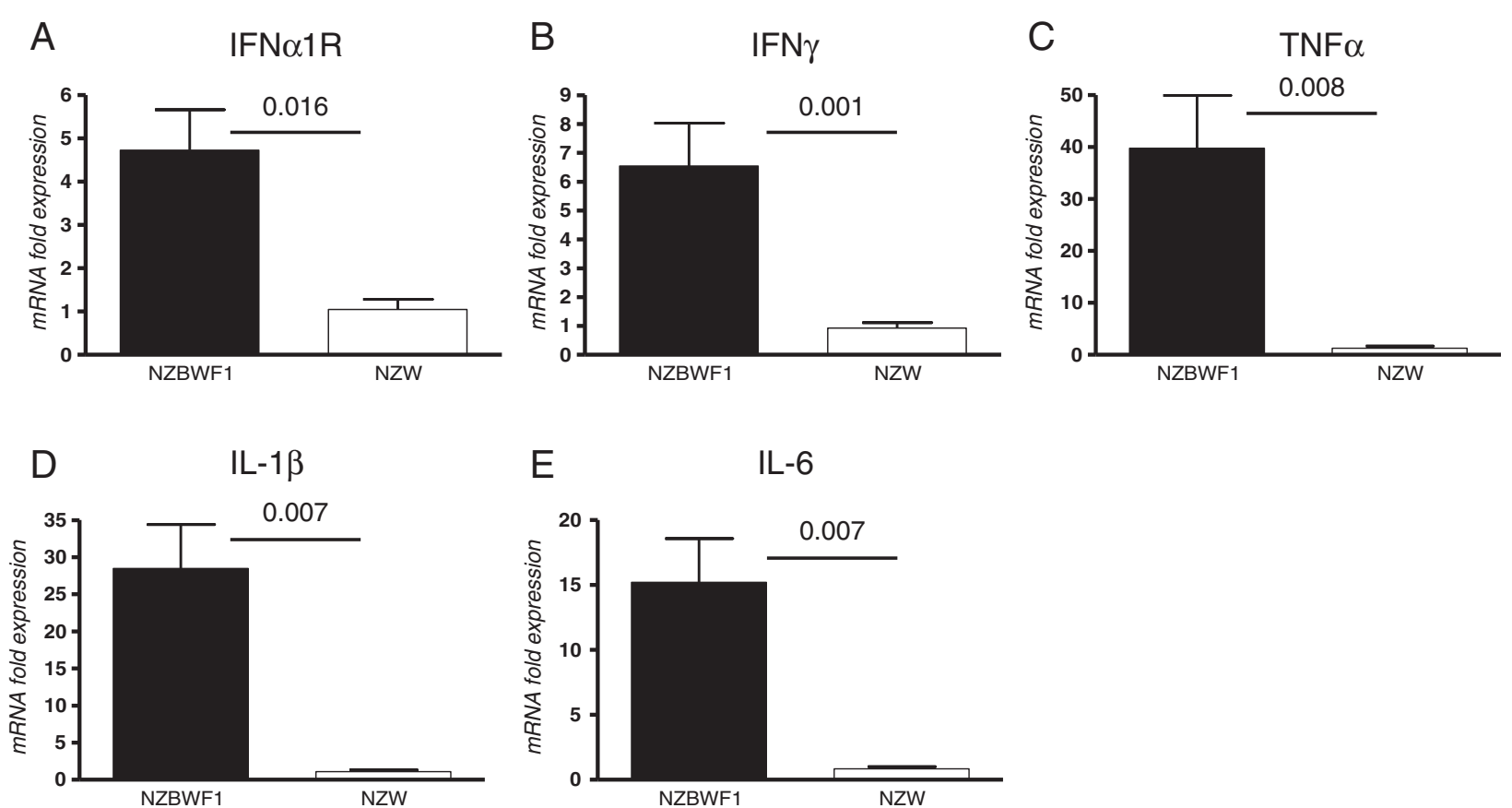

Figure 2 Upregulation of inflammatory markers in renal tissues from diseased New Zealand black $\mathbf{x}$ New Zealand white F1 (NZBWF1) mice. (A-E) Gene expression in kidneys of NZBWF1 mice relative to New Zealand white (NZW) control tissues was determined for the indicated markers by qRT-PCR using five mice per group. Statistical analysis was done using the Mann-Whitney test and $P$-values are indicated. 

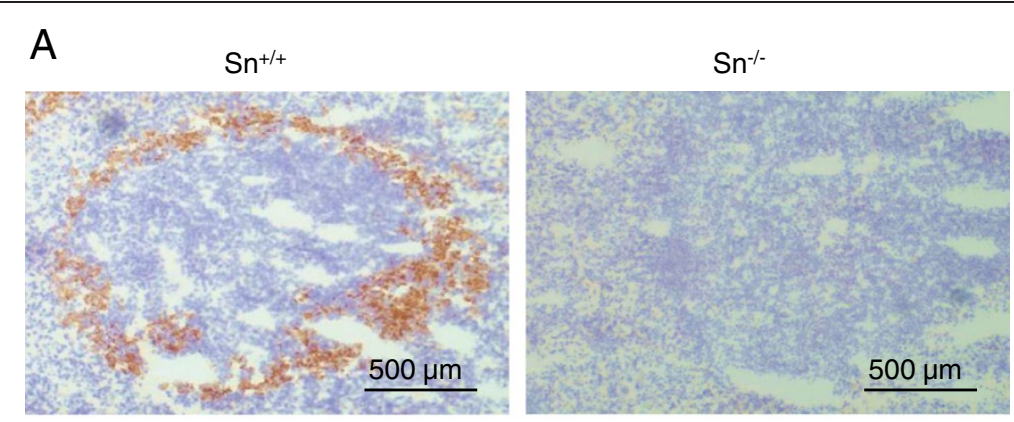

\section{B}

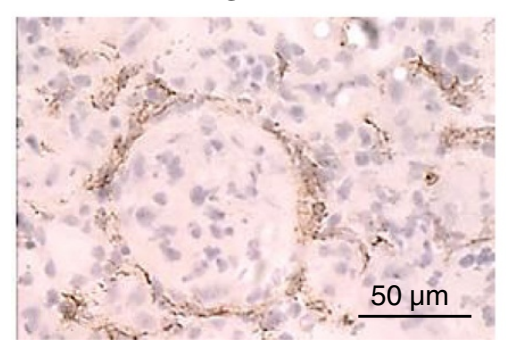

$\mathrm{Sn}^{-/-}$

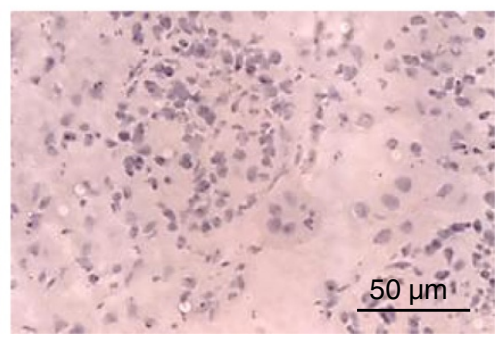

Figure 3 Generation of sialoadhesin (Sn)-deficient NZBWF1 mice. Frozen sections from spleen (A) and kidney (B) of Sn ${ }^{+/+}$and $\mathrm{Sn}^{-/-} \mathrm{NZBWF1}$ mice were stained for Sn using immunohistochemistry.

groups (Figure 4B). The severity of lupus nephritis was also assessed histologically using the World Health Organization (WHO) scoring system as outlined in Methods. There were no significant differences in glomerular, tubulointerstitial or perivascular scores between $\mathrm{Sn}^{-1-}$ and $\mathrm{Sn}^{+/+}$mice (Figure 4C). Taken together, these data demonstrated that $\mathrm{Sn}$ deficiency does not significantly influence the progression to end-stage nephritis in NZBWF1 mice.





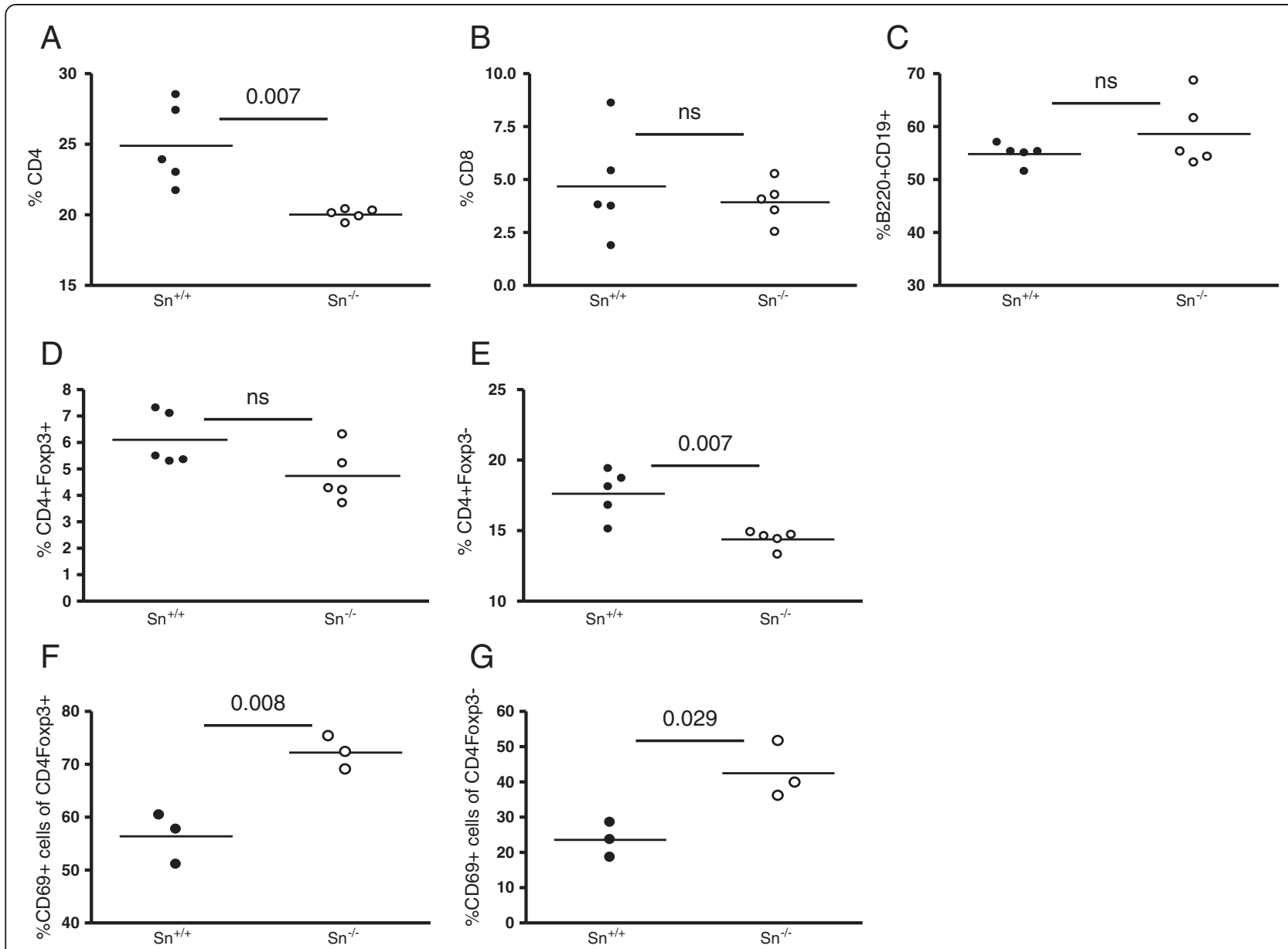

Figure 5 Flow cytometry of splenocytes from diseased sialoadhesin (Sn)+/+ and Sn-/- New Zealand black x New Zealand white F1 (NZBWF1) mice. (A-G) Splenocytes were isolated from 28-week-old diseased Sn+/+ (filled circles) and Sn-/- (open circles) NZBWF1 mice. Each circle represents an individual mouse. Statistical analysis was done using the Mann-Whitney test and $P$-values are indicated; ns, not significant.

\section{$\mathrm{Sn}^{-/-}$NZBWF1 mice have higher frequency of activated $\mathrm{CD}^{+} \mathrm{T}$ cells}

We have previously shown that $\mathrm{Sn}$ can interact with $\mathrm{Sn}$ ligands (SnL) induced on a subset of highly activated, potentially pathogenic $\mathrm{CD}^{+}{ }^{+}{ }^{-}$oxp $3^{-}$effector $\mathrm{T}$ cells (Teffs) and trigger their apoptosis [22]. SnL are also upregulated on subsets of $\mathrm{CD}^{+} \mathrm{FoxP}^{+}$regulatory $\mathrm{T}$ cells (Tregs) in experimental autoimmune encephalomyelitis (EAE), leading to an Sn-dependent reduction in numbers of Tregs and exacerbation of disease [17]. In order to examine the

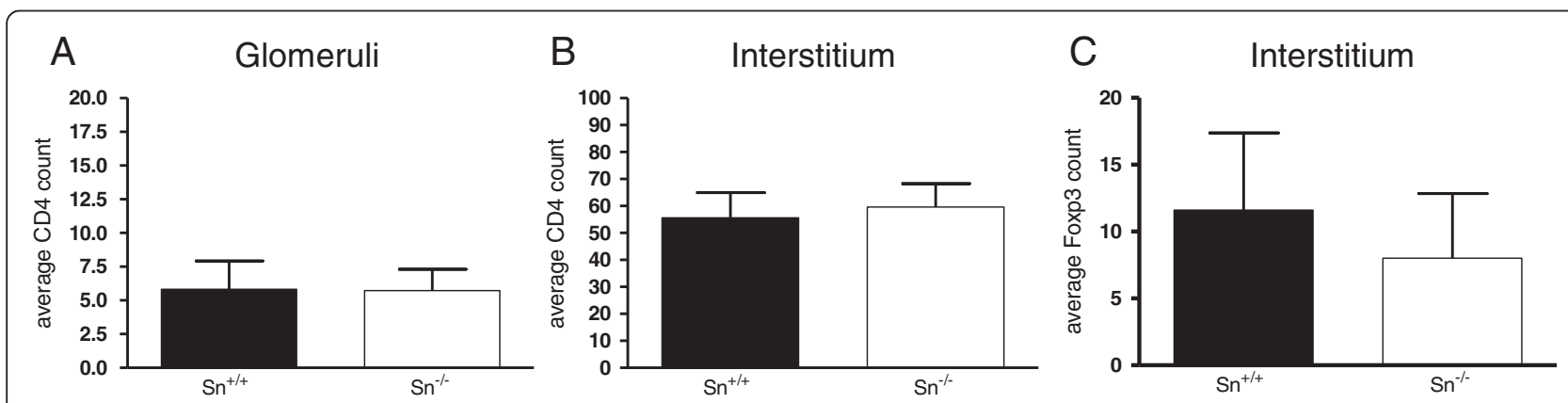

Figure 6 The frequency of CD4+ and Foxp3+ T cells in glomerular and tubulointerstitial compartments of sialoadhesin (Sn)+/+ and Sn-/- New Zealand black $x$ New Zealand white F1 (NZBWF1) mice. (A-C) Immunohistochemistry staining for CD4 and Foxp3 was done on renal sections from five mice per group. No significant differences were observed using the Mann-Whitney test. 
impact of Sn deficiency on different splenocyte subsets, we performed flow cytometric analysis (Figure 5). This revealed a significant reduction in the percentage of $\mathrm{CD}^{+}$ $\mathrm{T}$ cells in $\mathrm{Sn}^{-/-}$mice (Figure $5 \mathrm{~A}$ ), but there was no difference in $\mathrm{CD}^{+}$or $\mathrm{CD} 19^{+} \mathrm{B} 220^{+}$cells (Figure $5 \mathrm{~B}$ and $\mathrm{C}$ ). This reduction was observed in both Foxp $3^{+}$and Foxp3 $\mathrm{CD}^{+}$subsets (Figure 5D and E). However, the percentages of $\mathrm{CD}^{+}{ }^{+} \mathrm{Foxp}^{+}$and $\mathrm{CD} 4^{+}$Foxp3 $3^{-} \mathrm{T}$ cells that expressed the activation marker CD69 were both significantly increased in spleens of $\mathrm{Sn}^{-/-}$mice (Figure $5 \mathrm{~F}$ and $\mathrm{G}$ ). As activated $\mathrm{CD} 4^{+} \mathrm{T}$ cells express $\mathrm{SnL}$ [22], this is consistent with a negative regulatory role of $\mathrm{Sn}$ on both Teffs and Tregs in SLE.

In order to analyse the effect of Sn deficiency on $\mathrm{CD}_{4}^{+}$ $T$ cells in renal tissues of NZBWF1 mice, we examined the frequency of $\mathrm{CD}^{+}$and Foxp3 ${ }^{+} \mathrm{T}$ cells using immunohistochemistry. There were no differences in the frequency of $\mathrm{CD}^{+} \mathrm{T}$ cells in glomeruli and tubulointerstitial tissues between $\mathrm{Sn}^{+/+}$and $\mathrm{Sn}^{-/-}$mice (Figure 6A and $\mathrm{B}$ ). We were unable to demonstrate positive Foxp3 staining in the glomerular compartment, but the tubulointerstitial tissues showed similar numbers of Foxp3 $3^{+}$cells in $\mathrm{Sn}^{+/+}$and $\mathrm{Sn}^{-1-}$ mice (Figure 6C).

\section{Discussion}

The expression and function of $\mathrm{Sn}$ have been investigated in a number of inflammatory conditions in animal models and in humans [10,13-17,23-29]. The majority of these studies suggested a pro-inflammatory role for the molecule. Indeed, Sn deficiency in mice has been reported to be associated with disease amelioration in several autoimmune conditions [14-17]. Although increased Sn levels were found to be representative of disease activity in human SLE [12], it has remained unclear whether Sn plays a direct role in the disease pathogenesis. In this study, we found abundant $\mathrm{Sn}$ expression at the mRNA and protein levels in the kidneys of nephritic mice. In addition, a number of cytokines that have been implicated previously in the induction of Sn under inflammatory conditions were also upregulated [30]. As there was a parallel increase in gene expression of the pan macrophage markers CD68 and F4/80 in the nephritic mice, it is difficult to differentiate between an enhanced induction of $\mathrm{Sn}$ on renal macrophage subsets and generalised infiltration of kidney tissues by macrophages. However, immunohistochemistry analysis revealed that $\mathrm{Sn}^{+}$macrophages were mainly distributed in a periglomerular manner and absent from the glomerular tuft, unlike $\mathrm{CD}^{+} 8^{+}$macrophages. The significance of this preferential anatomical location of $\mathrm{Sn}^{+}$macrophages is currently unclear.

Previously, Sn expression on macrophages in a model of EAE was reported to downregulate numbers of $\mathrm{SnL}^{+} \mathrm{CD} 4^{+}$Foxp $^{+}{ }^{+}$Tregs via $\mathrm{Sn}-\mathrm{SnL}$ interactions, leading to increased disease severity [17]. Recently, we confirmed the abundance of $\mathrm{SnL}$ on Tregs and also identified a novel subset of activated CD4 ${ }^{+}$Foxp $3^{-}$Teffs bearing SnL following TCR ligation in vitro [22]. A phenotypically similar subset of $\mathrm{SnL}^{+} \mathrm{CD}^{+}{ }^{+}$Foxp3- Teffs was identified in proteinuric NZBWF1 mice, with the frequency of this subset correlating closely with disease status (proteinuria presence versus absence) [22]. We therefore hypothesized that Sn deficiency might be associated with an augmented disease severity due to an increase in these highly activated $\mathrm{CD} 4^{+}$Foxp $3^{-} \mathrm{T}$ cells. Surprisingly, however, despite an earlier onset of proteinuria, the disease progression was not different between $\mathrm{Sn}^{+/+}$and $\mathrm{Sn}^{-1-}$ mice. Since the frequency of both activated Teffs and Tregs was enhanced in the spleens of $\mathrm{Sn}^{-1-}$ mice, the net outcome on CD4 $\mathrm{T}$ cell function could be neutral and help explain why the severity of nephritis was similar regardless of the genotype. Importantly, our results indicate that unlike non-polygenic autoimmune conditions studied previously [14-17], Sn deficiency does not affect disease severity in SLE.

In conclusion, our findings suggest that $\mathrm{Sn}$ expression is increased in murine lupus nephritis and correlates with histological severity. However, Sn deficiency does not affect the disease progression or histological severity. Collectively, these data indicate that the role of $\mathrm{Sn}$ in SLE is redundant. As demonstrated by others [12,13], Sn serves as a potentially useful biomarker of inflammation and disease severity in SLE.

\section{Conclusions}

In the NZBWF1 model of murine lupus nephritis, Sn expression in the kidney was increased and correlated with histological severity of disease. Although Sn deficiency was associated with dynamic changes of splenic activated CD4 T cells and earlier onset of disease, it did not affect disease progression or histological severity. Collectively, these data suggest that $\mathrm{Sn}$ acts as a biomarker that is reflective of disease in murine lupus nephritis rather than playing a causal role.

\section{Additional file}

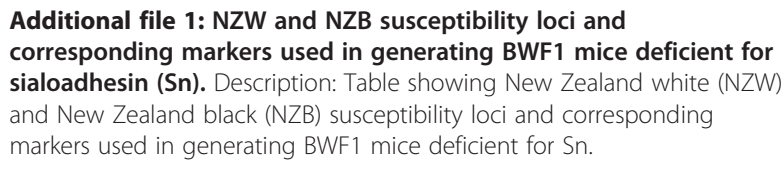

\section{Abbreviations}

BSA: Bovine serum albumin; EAE: Experimental autoimmune encephalomyelitis; EDTA: Ethylenediaminetetraacetic acid; ELISA: Enzyme-linked immunosorbent assay; FACS: Fluorescence-activated cell sorting; gcs: Glomerular cross-section; H\&E: Haematoxylin and eosin; HRP: Horse radish peroxidase; IFN: Interferon; Ig: Immunoglobulin; NZB: New Zealand black; NZBWF1: New Zealand black x New Zealand white F1; NZW: New Zealand white; PBS: Phosphate-buffered saline; PBST: Phosphate-buffered saline plus 0.05\% Tween; qRT-PCR: Quantitative reverse transcriptase-polymerase chain reaction; SLE: Systemic lupus erythematosus; Sn: Sialoadhesin; SnL: Sn ligands; Teffs: CD4 + Foxp3-effector 
T cells; TNF: Tumour necrosis factor; Tregs: CD4 + Foxp3+ regulatory T cells; WT: Wild-type.

\section{Competing interests}

The authors declare that they have no competing interests.

\section{Authors' contributions}

DK conceived, designed and performed experiments and wrote the first draft of the manuscript. HER conceived, designed and performed experiments. DK, HER and PRC contributed equally to data analysis and interpretation. PAL provided reagents, designed the genotyping protocol and contributed to drafting of the manuscript. PRC supervised the project and prepared the final manuscript. All authors read and approved the final manuscript.

\section{Acknowledgements}

This work was supported by a Wellcome Trust Clinical Fellowship 087078 (to DK) and Wellcome Trust Senior Fellowship 081882MA (to PRC). PAL was funded by a Wellcome Trust Programme Grant (083650/Z/07/Z). We thank the Renal Unit in Ninewells Hospital, Dundee, Scotland, UK, for their support with the immunohistochemistry analysis.

\section{Author details}

${ }^{1}$ Division of Cell Signalling and Immunology, College of Life Sciences, University of Dundee, Dow Street, Dundee DD1 5EH, United Kingdom. ${ }^{2}$ Cambridge Institute for Medical Research and Department of Medicine, University of Cambridge, Hills Road, Cambridge CB2 OXY, United Kingdom.

Received: 20 July 2013 Accepted: 11 October 2013

Published: 1 November 2013

\section{References}

1. Kotzin BL: Systemic lupus erythematosus. Cell 1996, 85:303-306.

2. Bruns A, Blass S, Hausdorf G, Burmester GR, Hiepe F: Nucleosomes are major $T$ and $B$ cell autoantigens in systemic lupus erythematosus. Arthritis Rheum 2000, 43:2307-2315.

3. Denny MF, Chandaroy P, Killen PD, Caricchio R, Lewis EE, Richardson BC, Lee KD, Gavalchin J, Kaplan MJ: Accelerated macrophage apoptosis induces autoantibody formation and organ damage in systemic lupus erythematosus. J Immunol 2006, 176:2095-2104.

4. Ren Y, Tang J, Mok MY, Chan AW, Wu A, Lau CS: Increased apoptotic neutrophils and macrophages and impaired macrophage phagocytic clearance of apoptotic neutrophils in systemic lupus erythematosus. Arthritis Rheum 2003, 48:2888-2897.

5. Feng $X$, Wu H, Grossman JM, Hanvivadhanakul P, FitzGerald JD, Park GS, Dong X, Chen W, Kim MH, Weng HH, Furst DE, Gorn A, McMahon M, Taylor M, Brahn E, Hahn BH, Tsao BP: Association of increased interferon-inducible gene expression with disease activity and lupus nephritis in patients with systemic lupus erythematosus. Arthritis Rheum 2006, 54:2951-2962

6. Baechler EC, Batliwalla FM, Karypis G, Gaffney PM, Ortmann WA, Espe KJ, Shark KB, Grande WJ, Hughes KM, Kapur V, Gregersen PK, Behrens TW: Interferoninducible gene expression signature in peripheral blood cells of patients with severe lupus. Proc Natl Acad Sci USA 2003, 100:2610-2615.

7. Crocker PR, Paulson JC, Varki A: Siglecs and their roles in the immune system. Nat Rev Immunol 2007, 7:255-266.

8. Klaas M, Oetke C, Lewis LE, Erwig LP, Heikema AP, Easton A, Willison HJ, Crocker PR: Sialoadhesin promotes rapid proinflammatory and type I IFN responses to a sialylated pathogen, Campylobacter jejuni. J Immunol 2012, 189:2414-2422.

9. Zou Z, Chastain A, Moir S, Ford J, Trandem K, Martinelli E, Cicala C, Crocker P, Arthos J, Sun PD: Siglecs facilitate HIV-1 infection of macrophages through adhesion with viral sialic acids. PLoS One 2011, 6:e24559.

10. York MR, Nagai T, Mangini AJ, Lemaire R, van Seventer JM, Lafyatis R: A macrophage marker, Siglec-1, is increased on circulating monocytes in patients with systemic sclerosis and induced by type I interferons and toll-like receptor agonists. Arthritis Rheum 2007, 56:1010-1020.

11. Delputte PL, Van Breedam W, Barbe F, Van Reeth K, Nauwynck HJ: IFN-alpha treatment enhances porcine Arterivirus infection of monocytes via upregulation of the porcine Arterivirus receptor sialoadhesin. J Interferon Cytokine Res 2007, 27:757-766.

12. Biesen R, Demir C, Barkhudarova F, Grun JR, Steinbrich-Zollner M, Backhaus M, Haupl T, Rudwaleit M, Riemekasten G, Radbruch A, Hiepe F, Burmester GR, Grutzkau A: Sialic acid-binding Ig-like lectin 1 expression in inflammatory and resident monocytes is a potential biomarker for monitoring disease activity and success of therapy in systemic lupus erythematosus. Arthritis Rheum 2008, 58:1136-1145.

13. Rose T, Grutzkau A, Hirseland H, Huscher D, Dahnrich C, Dzionek A, Ozimkowski T, Schlumberger W, Enghard P, Radbruch A, Riemekasten G, Burmester GR, Hiepe F, Biesen R: IFNalpha and its response proteins, IP-10 and SIGLEC-1, are biomarkers of disease activity in systemic lupus erythematosus. Ann Rheum Dis 2013, 72:1639-1645.

14. Jiang HR, Hwenda L, Makinen K, Oetke C, Crocker PR, Forrester JV: Sialoadhesin promotes the inflammatory response in experimental autoimmune uveoretinitis. J Immunol 2006, 177:2258-2264.

15. Ip CW, Kroner A, Crocker PR, Nave KA, Martini R: Sialoadhesin deficiency ameliorates myelin degeneration and axonopathic changes in the CNS of PLP overexpressing mice. Neurobiol Dis 2007, 25:105-111.

16. Kobsar I, Oetke C, Kroner A, Wessig C, Crocker P, Martini R: Attenuated demyelination in the absence of the macrophage-restricted adhesion molecule sialoadhesin (Siglec-1) in mice heterozygously deficient in P0. Mol Cell Neurosci 2006, 31:685-691.

17. Wu C, Rauch U, Korpos E, Song J, Loser K, Crocker PR, Sorokin LM: Sialoadhesin-positive macrophages bind regulatory $T$ cells, negatively controlling their expansion and autoimmune disease progression. J Immunol 2009, 182:6508-6516.

18. Oetke C, Vinson MC, Jones C, Crocker PR: Sialoadhesin-deficient mice exhibit subtle changes in B- and T-cell populations and reduced immunoglobulin M levels. Mol Cell Biol 2006, 26:1549-1557.

19. Wakeland E, Morel L, Achey K, Yui M, Longmate J: Speed congenics: a classic technique in the fast lane (relatively speaking). Immunol Today 1997, 18:472-477.

20. Nozaki Y, Yamagata T, Yoo BS, Sugiyama M, Ikoma S, Kinoshita K, Funauchi M, Kanamaru A: The beneficial effects of treatment with all-trans-retinoic acid plus corticosteroid on autoimmune nephritis in NZB/WF mice. Clin Exp Immunol 2005, 139:74-83.

21. Hadj-Slimane R, Chelbi-Alix MK, Tovey MG, Bobe P: An essential role for IFN-alpha in the overexpression of Fas ligand on MRL/lpr lymphocytes and on their spontaneous Fas-mediated cytotoxic potential. $J$ Interferon Cytokine Res 2004, 24:717-728.

22. Kidder D, Richards HE, Ziltener HJ, Garden OA, Crocker PR: Sialoadhesin ligand expression identifies a subset of CD4 + Foxp3- T cells with a distinct activation and glycosylation profile. J Immunol 2013, 190:2593-2602.

23. Xiong YS, Wu AL, Lin QS, YU J, Li C, Zhu L, Zhong RQ: Contribution of monocytes Siglec- 1 in stimulating $T$ cells proliferation and activation in atherosclerosis. Atherosclerosis 2012, 224:58-65.

24. Ashokkumar C, Gabriellan A, Ningappa M, Mazariegos G, Sun Q, Sindhi R: Increased monocyte expression of sialoadhesin during acute cellular rejection and other enteritides after intestine transplantation in children. Transplantation 2012, 93:561-564.

25. Bao G, Han Z, Yan Z, Wang Q, Zhou Y, Yao D, Gu M, Chen B, Chen S, Deng A, Zhong R: Increased Siglec-1 expression in monocytes of patients with primary biliary cirrhosis. Immunol Invest 2010, 39:645-660.

26. Xiong YS, Zhou YH, Rong GH, Wu WL, Liang Y, Yang ZX, Geng HL, Zhong RQ: Siglec-1 on monocytes is a potential risk marker for monitoring disease severity in coronary artery disease. Clin Biochem 2009, 42:1057-1063.

27. Ikezumi Y, Suzuki T, Hayafuji S, Okubo S, Nikolic-Paterson DJ, Kawachi H, Shimizu F, Uchiyama M: The sialoadhesin (CD169) expressing a macrophage subset in human proliferative glomerulonephritis. Nephrol Dial Transplant 2005, 20:2704-2713.

28. Hartnell A, Steel J, Turley H, Jones M, Jackson DG, Crocker PR: Characterization of human sialoadhesin, a sialic acid binding receptor expressed by resident and inflammatory macrophage populations. Blood 2001, 97:288-296.

29. Muerkoster S, Rocha M, Crocker PR, Schirrmacher V, Umansky V: Sialoadhesin-positive host macrophages play an essential role in graft-versus-leukemia reactivity in mice. Blood 1999, 93:4375-4386.

30. Taylor MW, Grosse WM, Schaley JE, Sanda C, Wu X, Chien SC, Smith F, Wu TG, Stephens M, Ferris MW, McClintick JN, Jerome RE, Edenberg HJ: Global effect of PEG-IFN-alpha and ribavirin on gene expression in PBMC in vitro. J Interferon Cytokine Res 2004, 24:107-118.

\section{doi:10.1186/ar4364}

Cite this article as: Kidder et al: Sialoadhesin deficiency does not influence the severity of lupus nephritis in New Zealand Black x New Zealand White F1 mice. Arthritis Research \& Therapy 2013 15:R175. 